\title{
Understanding the Need for Multiscaling in Biomaterial Mechanics
}

\section{Vikas Tomar}

Associate Professor, School of Aeronautics and Astronautics, Purdue University, West Lafayette, USA

Biological materials have evolved over millions of years and are often found as complex composites with superior properties compared to their relatively weak original constituents. The toughness of spider silk, the strength and lightweight of bamboos, self-healing of bone, high toughness of nacre, and the adhesion abilities of the gecko's feet are a few of the many examples of high performance natural materials. Hard biomaterials such as bone, nacre, and dentin have intrigued researchers for decades for their high stiffness, toughness, multifunctionality, and self-healing capabilities. For example, Nacre has 3000 times more toughness compared to its mineral constituent. Tooth enamel is 1000 times stiffer than its constituent protein polymer collagen. The general mechanical performance of these composites is quite remarkable. In particular, they combine two properties which are usually quite contradictory, but essential for the function of these materials. Bones, for example, need to be stiff to prevent bending and buckling, but they must also be tough since they should not break catastrophically even when the load exceeds the normal range. Such hard biological materials are not only light weight but also possess high toughness and mechanical strength.

One of the defining features of such biological composites is that they are highly hierarchical with different structures at different length scales. Often they are complex nanocomposites of soft fibrous polymeric phase and hard mineral phase. For instance, bone has up to seven levels of hierarchy and nacre shows up to six levels of hierarchical structure. Materials such as bone and nacre have such multi-level hierarchical structural design that concept of stress concentration at flaws remains invalid, leading to flaw tolerant structure. In spite of complex hierarchical structures, the smallest building blocks in such biological materials are at the nanometer length scale. For example, at the lowest level in bone, nanometer-sized crystals of carbonate apatite are embedded in the fibrous protein collagen in a well organized staggered arrangement.

In nanocomposite materials, the volume fraction of the proteinmineral interface can be enormous as the mineral bits have nanoscale size. For example, in a raindrop size volume of a nanocomposite, the area of interfacial region can be as large as a football field. Interfaces play crucial role in regulating the overall mechanical properties of nanocomposites. In case of hard biomaterials such as bone, dentin, and nacre, they have primarily an organic phase (e.g. tropocollagen (TC) or chitin) and a mineral phase (e.g. hydroxyapatite (HAP) or aragonite) arranged in a staggered arrangement. In bone, the crystalline mineral phase is preferentially aligned along the longitudinal axis of the polypeptide molecules permitting maximum contact area in a staggered arrangement. The extent of interfacial interaction and the interfacial arrangement are important determinants of the structure-function property relationship of biomaterials and influence the mechanical strength substantially. Such biological materials have been reviewed in appreciable detail, in the context of their hierarchical structure, material properties, and failure mechanisms.

An important aspect to focus in biomaterials engineering of hard biomaterials is the chemo-mechanics of the organic-inorganic interfaces and its correlation with overall mechanical behavior. This understanding is vital for selecting appropriate constituents, their size scales and their relative arrangements, which in turn is governed by the functional requirements of the composite materials. For example, a three dimensional (3D) explicit atomistic failure analyses of model Tropocollagen-Hydroxyapatite interfacial biomaterial (similar to material found in bone tissues) performed at the nanoscopic length scale, have pointed out that maximizing the contact area between the TC and HAP phases result in higher interfacial strength as well as higher fracture strength. Analyses have also shown that high toughness and strain hardening behavior of such biomaterial is due to reconstitution of columbic interactions between TC and HAP surfaces during interfacial sliding due to mechanical deformation. It has also been shown recently that changes in the residue sequences of TC molecules at the interface can affect the material mechanical strength considerably. Further, it has been shown earlier that moisture can play a major role in affecting the strength of such hybrid interfaces in biological materials.

Challenges lie in identifying nature's mechanisms behind imparting such properties and its pathways in fabricating and optimizing these composites. The route frequently acquired by nature is by embedding sub-micron or nano sized mineral particles in protein matrix in a well organized hierarchical arrangement. The key here is the formation of large amount of precisely and carefully designed organic-inorganic interfaces and synergy of mechanisms acting over multiple scales to distribute loads and damage, dissipate energy, and resist change in properties owing to damages such as cracking.
*Corresponding author: Vikas Tomar, Associate Professor, School of Aeronautics and Astronautics, Purdue University, West Lafayette, USA, Tel: 765-4943-006; Fax: 765-4940-307; E-mail: tomar@purdue.edu

Received August 22, 2012; Accepted August 22, 2012; Published August 24 2012

Citation: Tomar V (2012) Understanding the Need for Multiscaling in Biomaterial Mechanics. J Nanomed Nanotechol 3:e113. doi:10.4172/2157-7439.1000e113

Copyright: () 2012 Tomar V. This is an open-access article distributed under the terms of the Creative Commons Attribution License, which permits unrestricted use, distribution, and reproduction in any medium, provided the original author and source are credited. 